\title{
Prediction of Neoadjuvant Chemotherapy Outcome in Breast Cancer Patients
}

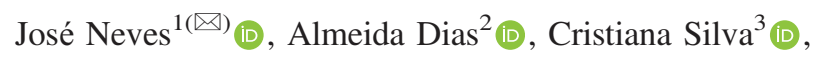

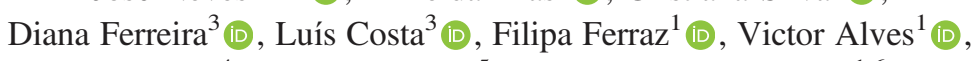 \\ João Neves ${ }^{4}$ (1), Jorge Ribeiro ${ }^{5}$ (1), and Henrique Vicente ${ }^{1,6}$ (B) \\ ${ }^{1}$ Centro Algoritmi, Universidade Do Minho, Braga, Portugal \\ \{jneves,valves\}@di.uminho.pt, filipatferraz@gmail.com \\ 2 CESPU, Instituto Universitário de Ciências Da Saúde, Gandra, Portugal \\ a.almeida.dias@gmail.com \\ 3 Departamento de Informática, Escola de Engenharia, Universidade Do Minho, \\ Braga, Portugal \\ \{a71665,a72226,a66516\}@alunos.uminho.pt \\ ${ }^{4}$ Mediclinic Arabian Ranches, PO Box 282602, Dubai, United Arab Emirates \\ joaocpneves@gmail.com \\ 5 ARC4DigiT - Applied Research Center for Digital Transformation, \\ Escola Superior de Tecnologia E Gestão, \\ Instituto Politécnico de Viana Do Castelo, Viana Do Castelo, Portugal \\ jribeiro@estg.ipvc.pt \\ ${ }^{6}$ Departamento de Química, Escola de Ciências E Tecnologia, \\ Centro de Química de Évora, Universidade de Évora, Évora, Portugal \\ hvicente@uevora.pt
}

\begin{abstract}
Breast Cancer is the most common invasive cancer in women worldwide. Indeed, it is imperative to investigate which factors influence the development of this disease in order to improve the efficiency of the treatment and to allow for a balanced follow-up. In fact, this article has in mind an original Case Based Reasoning (CBR) approach to problem solving, complemented with a novel approach to Knowledge Representation and Reasoning that takes into consideration the data items entropic states. It works towards cancer's assessment after Neoadjuvant Chemotherapy in terms of its size, shape, and texture.
\end{abstract}

Keywords: Breast Cancer - Neoadjuvant Chemotherapy ·

Knowledge Representation and Reasoning - Case Based Reasoning • Entropy • Logic Programming 